\title{
CONF-9608173--1
}

\section{COULOMB EXCITATION OF RADIOACTIVE NUCLEAR BEAMS IN INVERSE KINEMATICS}

\author{
N. V. ZAMFIR $a, b, c, d$, C.J. BARTON ${ }^{c}$, R.F, CASTEN ${ }^{a, b}$, \\ R.L. GILL ${ }^{a}$, D.S. BRENNER ${ }^{c}$, and A. ZILGES ${ }^{b, e}$ \\ a Brookhaven National Laboratory, Upton, NY 11979, USA \\ - WNSL, Yale University, New Haven, CT 06520, USA \\ c Clark University, Worcester, MA 01610, USA \\ dInstitute of Atomic Physics, Bucharest Magurele, Romania \\ ' Institut für Kernphysik der Universität zu Köln, Germany
}

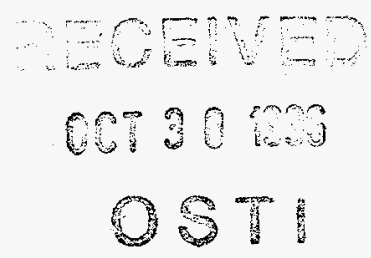

Techniques for the measurement of $B\left(E 2: 0_{1}^{+} \rightarrow 2_{1}^{+}\right)$values by Coulomb excitation of Radioactive Nuclear Beams in inverse kinematics are described. Using a thin, low $Z$ target, the Cuulumb excited beam nuclei will decay in flight duwnstream of the target. For long lifetimes (nanosecond range) these nuclei decay centimeters downstream of the target and for shorter lifetimes (picoseconds or less) they decay near the target. Corresponding to these two lifetime regimes two methods have been developed to measure $\gamma$ rays from the Coulomb excited nuclei: the lifelime method in which the lifetime of the excited state is deduced from the decay curve and the integral method in which the $B(E 2)$ value is extracted from the measured total Coulomb excitation cross section.

\section{Introduction}

The advent of Radioactive Nuclear Beams (RNB) facilities will soon open new perspectives for nuclear structure studies. However, they will never provide the wealth of data we are accustomed to in nuclear spectroscopy. Consequently, the experiments at these facilities must provide a very high degree of selectivity and sensitivity to structural features. Recent studies 1.2 have shown that the knowledge of the energies of the first excited states together with the related $B(E 2)$ values are sufficient to disclose many aspects of nuclear structure.

An ideal way to determine the properties of the first exited state is low energy Coulomb excitation. The simplicity of the $\gamma$ ray spectrum obtained allows one to use lower resolution but higher efficiency $\mathrm{NaI}(\mathrm{Tl})$ detectors to compensate for low intensities of RNBs. Futhermore, the consequences of the beam radioactivity can be minimized by using inverse kinematics on low $\mathrm{Z}$ targets, when the Coulomb excited beam nuclei exit the target in a narrow cone and decay in flight. Corresponding to the range of the lifetime of the Coulomb excited state, two methods were established: a lifetime method for the ns range and an integral method for lifetimes $\sim$ few 100 ps or less. The methods, described below, were tested by simulating the conditions (beam intensity and energy) expected for an RNB facility by using the ESTU Tandem

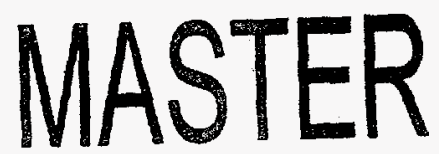


Van de Granff accelerator of the Yale University Wright Nuclear Structure Laboratory, to provide beams of ${ }^{188} \mathrm{O}$ s and ${ }^{192} \mathrm{O}$ s with energies of $\sim 270 \mathrm{MeV}$. These beams are good test cases since the $2_{1}^{+}$mean lifetimes have convenient values for the two methods: 0.99 ns for ${ }^{188} \mathrm{O}$ s and 0.42 as for ${ }^{192} \mathrm{Os}_{3}$

\section{Lifetime Method}

If the lifetime of the Coulomb excited state is on the order of $1 \mathrm{~ns}$, and the nucleus has an exit velocity of $\sim 5 \%$ c (corresponding to a beam energy of $1.5 \mathrm{MeV} / \mathrm{A}$ ), the decays will occur downgtream of the target over a range of several centimeters. By using a linear array of $\mathrm{Nal}(\mathrm{Tl})$ detectors (see fig.1), the decay curve for the excited state can be determined from measuring the relative number of decays seen by each (further in distance downstream, successively later in time) detector. Each detector is shielded so as to see only a narrow "time window" along the flight path. If the array covers a distance corresponding to a few lifetimes it will, in effect, measure the lifetime of the excited state, and we call the method the lifetime method.

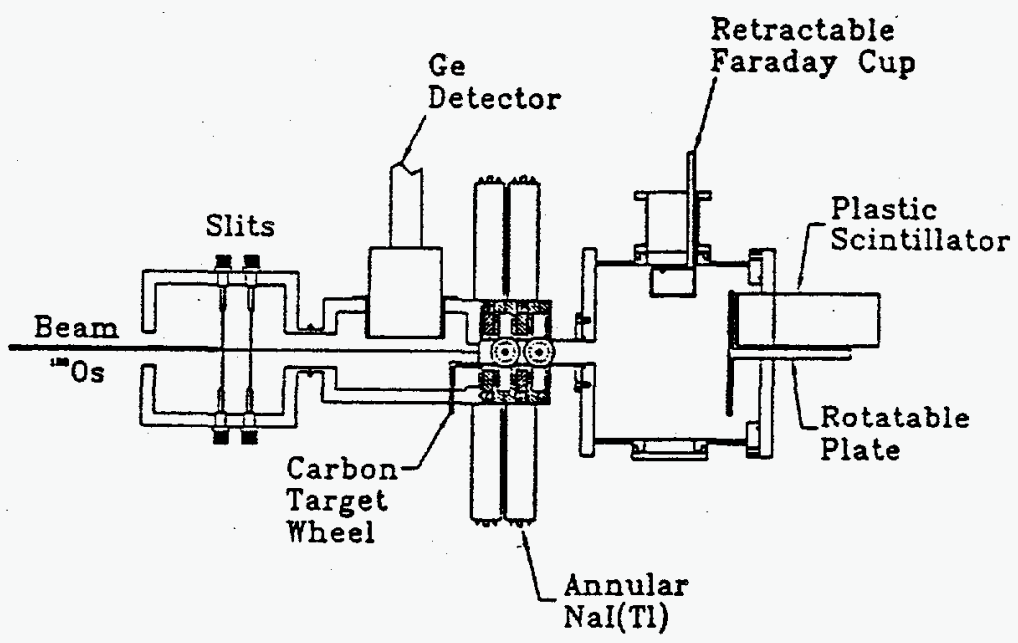

Figure 1: Diagram of the apparatus used in the Coulomb excilation in inverse kinematics experiments using the lifetime method. 
A detector located at a position downstream of the target records a total number of counts, $C$, such that:

$$
C=\iint d N(t, \Omega) \epsilon(t, \Omega), d \Omega d t
$$

where the integral is over the volume defined by each detector's collimating geometry. The time of flight, $t$, measured from the target, is determined from the effective position of the detectors that record the decay of the excited particle and the exiting velocity. The effective position is determined by an iterative method described below and the velocity is measured with a Ge detector by taking advantage of the Doppler shift of $\gamma$ rays from the decay in flight.

The absolute detection efficiency, $\epsilon(t, \Omega)$ for $\gamma$ rays emitted is established either by measurement or by calculation. The efficiency along the apparatus centerline can be determined with a calibrated source, positioned at successive points downstream of the target. However, this only gives an estimate of the true efficiency, since the activity is actually distributed (non-uniformly) throughout a cone. Taking advantage of the fact that with this method only the relative number of counts are important, it is simpler to consider only the geometrical efficiency and to calculate it using a Monte Carlo technique, by randomly choosing a large number of scattering angles and $\gamma$ ray emission angles, weighted by the angular distribution of Coulomb excited nuclei, and the angular distribution of the emitted $\gamma$ rays. Relativistic effects were also considered (see ref. ${ }^{3}$ ).

The number of Coulomb excited nuclei, $d N(t, \Omega)$, that scatter into, and decay in, a particular volume of space is defined as:

$$
d N(t, \Omega)=\lambda N_{0} e^{-\lambda t} d P(\Omega)
$$

where $\lambda$ is the decay constant, $N_{0}$ is the total number of Coulomb excited nuclei produced at the target, and $d P(\Omega)$ is the probability for a Coulomb excited nucleus to be scattered into a solid angle $\Omega$ :

This probability can be calculated using the relation:

$$
d P=\frac{1}{\sigma} \frac{d \sigma}{d \Omega}
$$

where $\sigma$ is the total Coulomb excitation cross section and $d \sigma / d \Omega$ is the differential scattering cross section.

The integral over the solid angle in eq. (1) yields the weighted geometric efficiency of the detector, $w(t)$, and eq. (1) can be written as: 


$$
C=\lambda N_{0} \int_{a}^{b} e^{-\lambda t} w(t) d t
$$

where $a$ and $b$ are the lower and upper limits of the field of view of each detector along the time axis, repectively.

By using the First Mean Value Theorem for integrals eq. (4) becomes:

$$
C=\lambda N_{0}[b-a] w\left(t_{\text {mean }}\right) e^{-\lambda t_{\text {mes }}}
$$

where $0<t_{\text {mean }}<(a+b) / 2$ and depends on $\lambda$.

Assuming that $t_{\text {mean }}$ is known for each detector, the new equation can be used to determine $\lambda$ by fitting the data (the measured values of $\mathrm{C}$ for each detector) to:

$$
\ln \frac{C}{[b-a] w\left(t_{\text {mean }}\right)}=-\lambda t_{\text {mean }}+\text { const. }
$$

To determine $t_{\text {mean }}$ for each detector an iterative technique is used. By using for $t_{\text {mean }}$ the value $t_{\text {center }}=[a+b] / 2$ from eq. (6) a seed value for $\lambda$ is determined. With the seed value, $\lambda_{s e e d}$, and using the Mean Value Theorem, a new $t_{\text {mean }}$ is determined from

$$
\int_{a}^{b} e^{-\lambda_{\text {seed }} t} w(t) d t=[b-a] w\left(t_{m e a n}\right) e^{-\lambda_{\text {sced }} t_{\text {mean }}}
$$

Once $t_{\text {mean }}$ has been determined for each detector, a fit can now be made to eq. (6). The decay constant, $\lambda$, generated from this fit is the new seed for the next iteration. The steps described above are repeated until $\lambda$ converges to a stable value (practically, only a few iterations are necessary).

The method was tested with a ${ }^{188} \mathrm{Os}$ beam of $270 \mathrm{MeV}$. Figure 2 shows the spectra obtained by placing three semi-annular $\mathrm{NaI}(\mathrm{Tl})$ detectors at successive positions along the beam line. The decay of the ${ }^{188} \mathrm{Os}_{2}^{+}$level at $155 \mathrm{keV}$ with distance from the target is clearly observed. The area of the $155 \mathrm{keV} \gamma$ ray peak versus time, corrected for the response of the detector array, is shown in fig.3. The line represents the best fit to the data and corresponds to a lifetime (mean life) for the $2_{1}^{+}$state of $1.03 \pm 0.05 \mathrm{~ns}$, as compared to the accepted value of $0.99 \pm 0.03 \mathrm{~ns}^{4}$.

\section{Integral Method}

If the lifetime is short relative to the flight time (picoseconds or less), the B(E2) value can be determined by measuring the total number of decays and deducing 


\section{DISCLAIMER}

Portions of this document may be illegible in electronic image products. Images are produced from the best available original document. 


\section{DISCLAIMER}

This report was prepared as an account of work sponsored by an agency of the United States Government. Neither the United States Government nor any agency thereof, nor any of their employees, makes any warranty, express or implied, or assumes any legal liability or responsibility for the accuracy, completeness, or usefulness of any information, apparatus, product, or process disclosed, or represents that its use would not infringe privately owned rights. Reference herein to any specific commercial product, process, or service by trade name, trademark, manufacturer, or otherwise does not necessarily constitute or imply its endorsement, recommendation, or favoring by the United States Government or any agency thereof. The views and opinions of authors expressed herein do not necessarily state or refleet those of the United States Government or any agency thereof. 


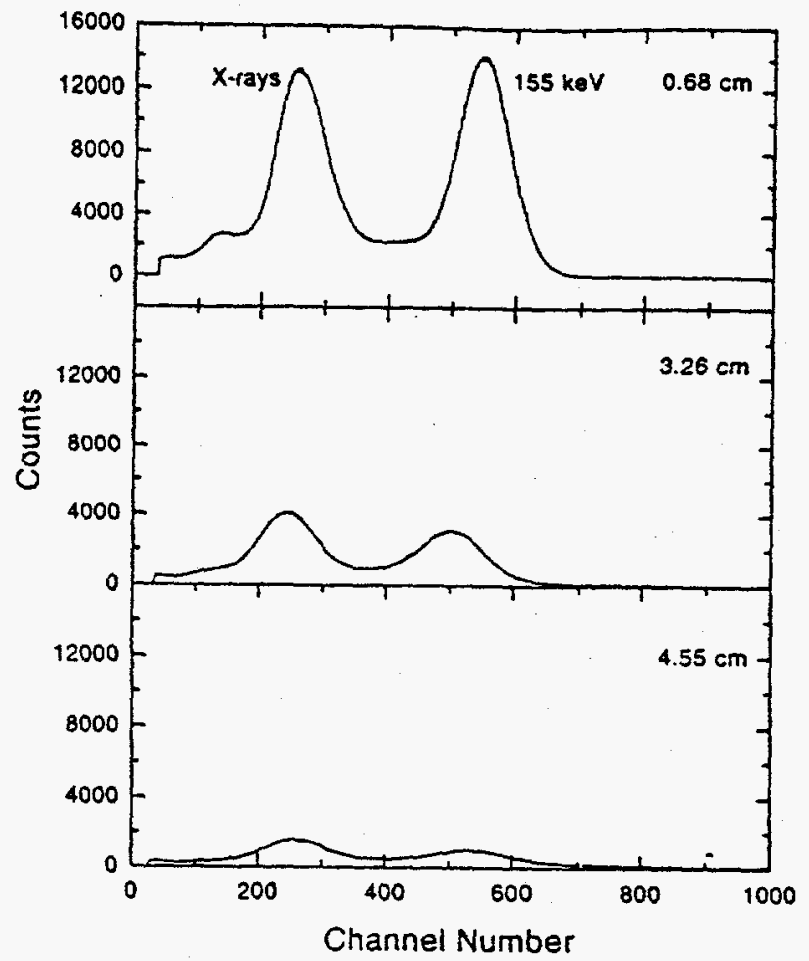

Figure 2: Spectra from Coulomb excited ${ }^{180} \mathrm{O}$ s nuclei, obtained with the linear semi-annular $\mathrm{NaI}(\mathrm{II})$ detector array. Each spectrum, from the top down, shows the results from a successively later time window. The distance from the target to the center of the detector is given in the upper right hand corner of each spectrum.

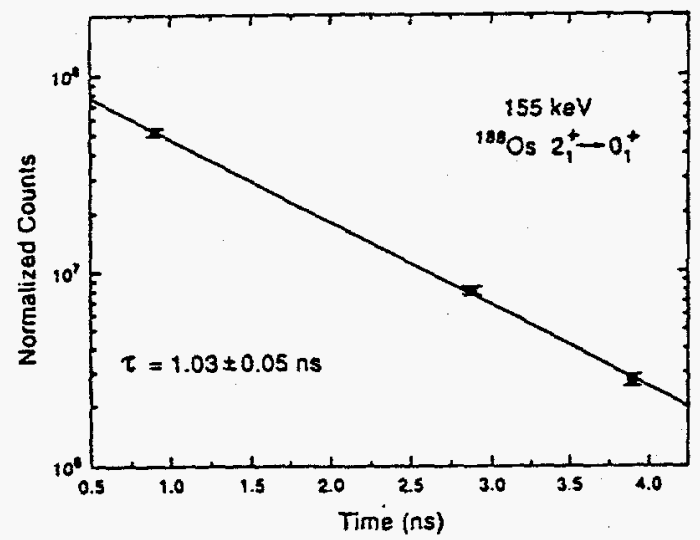

Figure 3: Decay curve for the $2_{1}^{+} \rightarrow 0_{1}^{+} 155 \mathrm{keV}$ transition in ${ }^{188} \mathrm{O}$, including corrections for relative efficiency. The line represents the best fit to the dats. 
the reaction cross section. For such measurements, we use a "through-well" $\mathrm{NaI}(\mathrm{Tl})$ detector which replaces the array of the $\mathrm{NaI}(\mathrm{Tl})$ detectors. The target is near its geometric center and the geometric efficiency is $\sim 90 \%$. This method, called the integral method, requires a knowledge of the entry and exit beam energy from the target, the incident beam intensity, and the absolute efficiency of the detector. The exiting energy can be measured directly by observing the Doppler shift of $\gamma$ rays from excited states that decay in flight.

A consequence of the high geometric efficiency of the through-well detector for a source that is near its geometric center is that the detector can observe transitions which occur in coincidence and will sum the energies of these into a single peak. In order to correctly calculate the absolute efficiency for detecting a $\gamma$ ray, the areas for all the sum peaks that subtract intensity from that expected for that $\gamma$ peak in the absence of summing must be considered.

The method was tested with ${ }^{192} \mathrm{Os}$ beam at $270 \mathrm{MeV}$. The spectrum obtained is shown in fig. 4. The $B\left(E 2 ; 0_{1}^{+} \rightarrow 2_{1}^{+}\right)$value measured in this experiment is $1.97 \pm 0.16 e^{2} b^{2}$, which agrees well with the accepted value of $2.05 \pm 0.07 e^{2 b^{2}}$.

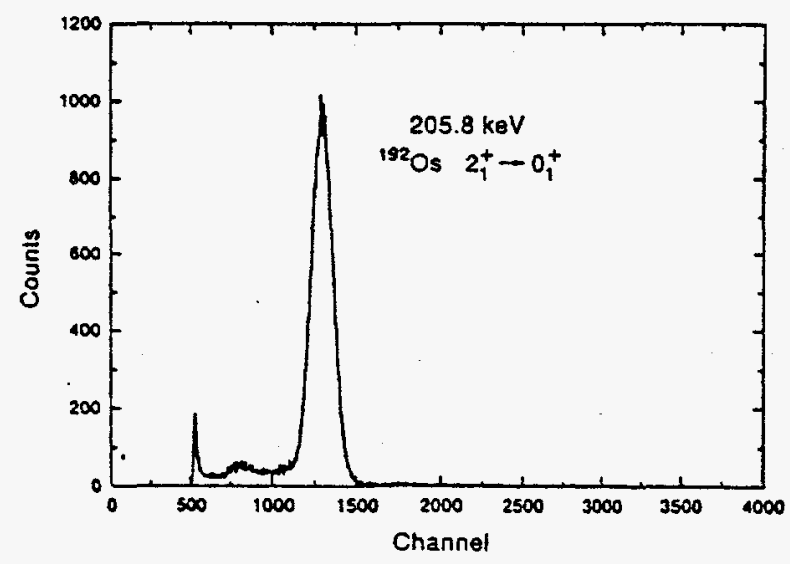

Figure 4: A spectrum from the decay of Coulomb excited ${ }^{192} \mathrm{O}$, taken with the through-well $\mathrm{NaI}(\mathrm{II})$ detectur.

Very recently a test of this method with an RNB of ${ }^{108} R u$ ions was carried out at the National Supereonducting Cyclotron at Michigan State University. Preliminary analysis shows that, with only a few hundred ${ }^{108} R u$ ions/sec. the $2_{1}^{+} \rightarrow 0_{1}^{+} \gamma$-ray was observed in a relatively short run. 


\section{Other Techniques}

In many deformed nuclei, conversion electron emission competes with, and sometimes dominates, $\gamma$ ray emission for the $2_{1}^{+} \rightarrow 0_{1}^{+}$transition. A linear array of Si PIN photodiodes can thus be used, employing the same principles as the $\mathrm{NaI}(\mathrm{Tl})$ array, to determine the $B\left(E 2 ; 0_{1}^{+} \rightarrow 2_{1}^{+}\right)$from the lifetime. Four $7.5 \mathrm{~mm} \times 20 \mathrm{~mm}$ Si PIN diodes (Hamamatsu S2662 series) were placed in a special beam tube, substituted for the NaI(Tl) array tube. The spectra obtained in 5 hours with an ${ }^{188} \mathrm{O}$ s beam intensity of $\sim 10^{9}$ nuclei/sec. are shown in fig. 5. The $K$ and $L+M$ lines associated with the decay of the 155 $\mathrm{keV} 2_{1}^{+} \rightarrow 0_{1}^{+}$transition are clearly evident.

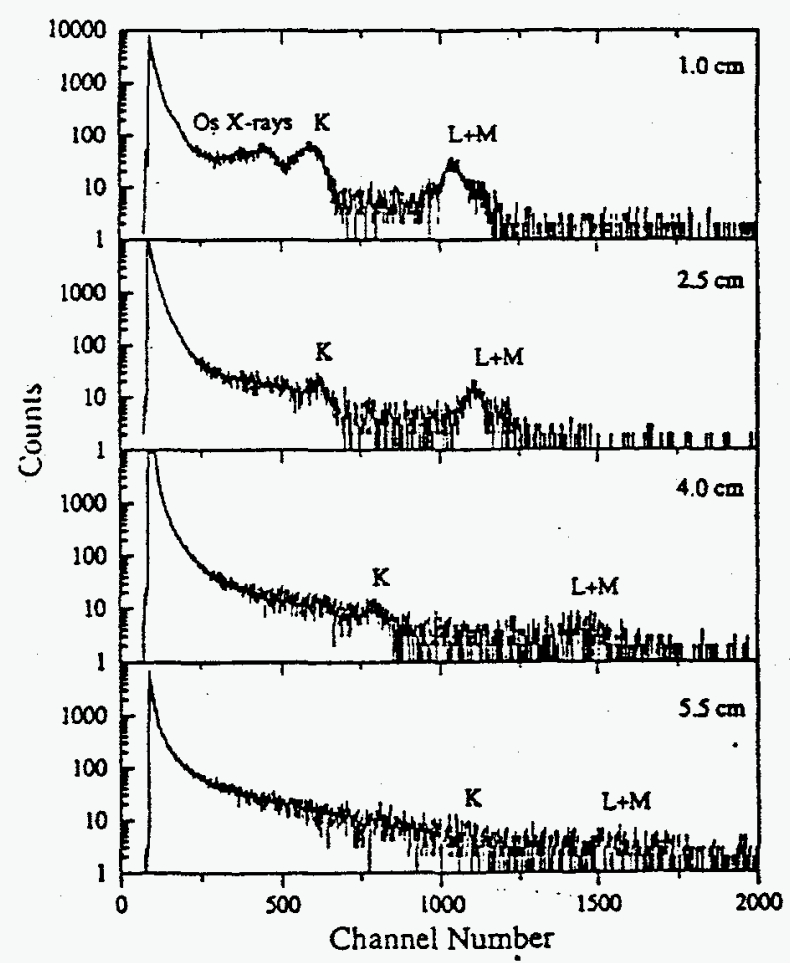

Figure 5: Conversion electron spectra obtained trom the decay of Coulomb excited ${ }^{180} \mathrm{Os}$ measured with a four detector Si PIN dirde array. The $K$ an $L+M$ lines for the $155 \mathrm{keV}$ $\left(2_{1}^{+} \rightarrow 0_{1}^{+}\right)$transition are indicated in each spectrum. The number in the upper right enrner of each spectrum gives the distance from the target to the center of each detector. 
The lifetime of the Coulomb excited state is deduced from the decay curve obtained using the Si PIN diodes array, in a similar way as the lifetime method using the $\mathrm{NaI}(\mathrm{Tl})$ detectors array.

Another technique that was tested involved measuring the intensity ratio of Doppler shifted to unshifted $\gamma$ rays. The technique is similar to the Recoil Distance (plunger) method, except that here the stopper travels over a distance of several cm, and is limited to lifetimes in the ns regime.

\section{Conclusion}

As RNB facilities become available, Coulomb excitation in inverse kinematics will prove to be a valuable technique for mapping the evolution of nuclear structure into new mass regions. The lifetime method is useful for directly measuring the decay curve for a Coulomb excited state with the lifetime in the nanosecond range. The use of a high efficiency $\mathrm{NaI}(\mathrm{Tl})$ linear array will make it possible to measure $B(E 2)$ values, at moderate beam intensities, $\left(\sim 10^{6}\right.$ nuclei/sec), in a day or two.

The integral method, using the through-well Nal(Tl) detector, is applicable to shorter lifetimes, and is a high efficiency method for lower beam intensities, perhaps as low as $10^{3}$ nuclei/sec. This latter feature is especially helpful at fragmentation RNB facilities, where high intensities may be difficult to obtain. It also raises the possibility of studying Coulomb excitation of nuclei after separation through a mass analyzer, using the RNB to bombard a stable beam, and to produce even more exotic nuclei.

Two other techniques, conversion electron detection and a Doppler shift ratio method, were developed. The initial results are promising, and development of both methods is continuing.

\section{Acknowledgments}

This work was supported under Contracts DE-AC02-76CH00016, DE-FG0288ER40417, and DE-FG02-91ER40609 with the U.S. Department of Energy.

1. R.F. Casten and N.V. Zamfir, Phys. Rev. Lett. 70 (1993) 402

2. N. V. Zamfir, G. Hering, R.F. Casten, and P.Paul, Phys. Lett. B357 (1995) 515

3. C.J. Barton et al, Nucl. Instrum. and Methods (submitted).

4. S. Raman et al, At.Data and Nucl.Data Tables 36 (1987) 1 